\title{
Model-based Estimation of Molten Metal Analysis in the LD Converter: Experiments at SSAB Tunnplåt AB in Luleå
}

\author{
Wolfgang Birk ${ }^{\star \dagger}$, Andreas Johansson ${ }^{\dagger}$, Alexander Medvedev ${ }^{\dagger}$, and Robert Johansson ${ }^{\ddagger}$ \\ ${ }^{\star}$ Corresponding author: Tel. +46 920 91907,Fax +46 920 91558, eMail wolfgang@ @m.luth.se \\ ${ }^{\dagger}$ Control Engineering Group, Luleå University of Technology, SE-971 87 Luleå, Sweden \\ ${ }^{\ddagger}$ SSAB Tunnplåt AB, SE-971 88 Luleå, Sweden
}

\begin{abstract}
Experiences from field tests of a model-based molten metal analysis estimation system for the LD converter process are reported. Experiments have been carried out during a six-months long period on two converters at SSAB Tunnplåt $\mathrm{AB}$ in Luleå, Sweden. The achieved results prove viability of the approach taken and indicate its high potential regarding estimation accuracy and robustness. It is also concluded that some further system development is necessary to enable modeling of additives and lance level before the system can be recommended for permanent installation.
\end{abstract}

\section{BACKGROUND}

This article is a follow-up of a presentation at the IAS2000 in Rome [1] describing the principles behind a new model-based system for real-time estimation of carbon and silicon content in the basic oxygen steelmaking process. During 2001, extensive field tests of the system have been carried out in cooperation with SSAB Tunnplåt $\mathrm{AB}$ in Luleå and SSAB Oxelösund $\mathrm{AB}$ in Oxelösund. It is well known that advanced model-based estimation techniques require extensive implementation and validation efforts until they become useful in an industrial environment. Thus, the main objective of the tests has been to evaluate estimation accuracy and robustness under realistic process conditions and collect reliable data for further system development. In this paper, the experimental results and practical experiences from a large number of converter heats at plant in Luleå are analyzed and reported.

\section{A. LD converter process}

The top blown basic oxygen method has been developed 50 years ago and is employed to reduce by oxidation the contents of carbon, silicon and other contaminating components in the hot metal from the blast furnace. The converter process is run as follows. The metal scrap, hot metal and slag-making substances are loaded in the converter. Other additives such as ferrosilicon can be provided later in the process. Oxygen $\left(\mathrm{O}_{2}\right)$ is blown through a lance at a supersonic speed onto the metal surface and does oxidize the metal components, mainly iron $(\mathrm{Fe})$, silicon $(S i)$, manganese $(M n)$, and carbon $(C)$. The oxides, together with metal droplets, form a foaming slag, in which more carbon will react with the oxides and produce carbon monoxide $(C O)$. In combination with oxygen, some of the carbon monoxide will produce carbon dioxide $\left(\mathrm{CO}_{2}\right)$.

\section{B. Process control}

The converter is controlled by an operator, who judges the state of the process based upon a number of measurements, e.g. a sound level measurement obtained by a sonicmeter and analysis of the off-gas [2]. The heat is completed when the content of carbon in the metal is considered to be ordered one. The operator may also visually monitor the flame drop above the converter mouth. The quality of the final product is therefore highly dependent on the experience and the judgment of the individual operator.

A major difficulty in the way of controlling the converter process is the lack of a reliable measurement of the steel analysis. Instead, different kinds of estimation techniques are used.

An important metallurgical process variable of the converter process is decarburization rate, i.e. the rate at which carbon is oxidized. Some of the steel mills apply simple empirical models for decarburization rate estimation based on the off-gas analysis, other take advantage of more advanced identified dynamic models, e.g. in the form of neural networks, for different process stages and conditions. An example of a data-driven system based on a combined technique is MEFCON by Mefos [3] where both physical static mass balance and estimated dynamic process models fed by real-time measurements of the off-gas analysis and gas flows are incorporated.

By means of the measured off-gas analysis and flow, MEF$\mathrm{CON}$ recalculates at each sampling instant the mass balance equations of the process until a relatively low threshold carbon content value is hit by the estimate. Then, a first-order dynamic model is activated to follow the decarburization process until the blow is over.

Under steady process conditions and provided accurate sensory data, this approach has been shown to produce satisfactory results [4].

\section{EstimATION TECHNIQUE}

To deliver a more viable, from a control point of view, solution to the molten metal analysis estimation problem, a so-called observer technique has been chosen.

The purpose of the observer is to project sensory data onto a mathematical model of the process, thus separating the measurements in two parts - one comprising the data complying with the model dynamics and another, usually called residual, including instances generated by model uncertainty and disturbances. In the observer, the process model is conventionally described by a system of differential equations which form as well enables estimation of otherwise unmeasurable process variables. The residual is fed back to the process model in order 
to guarantee observer stability and enhance convergence of the estimates. This is very much similar to the prediction-correction algorithms used in numerical integration. However, the actual process dynamics are known only approximately.

An observer comprised of a non-linear simplified mathematical model of the LD converter [2] and a non-linear feedback is employed in order to obtain real-time estimates of the molten metal analysis. Measurements of the oxygen flow through the lance as well as the analysis and the flow of the off-gas are used for updating the process model. The observer design method is based on evaluating the sensitivity functions of the process output signals, $i$. $e$. the off-gas analysis, with respect to the process states, $i$. $e$. the carbon and silicon content, [5].

The model of the derived LD converter is based on mathematical description of the physical and chemical processes taking place in the process during a heat. Therefore, the process model is independent of the actual converter individual it works with. Of course, this is achieved at cost of quite coarse description of the physical and chemical phenomena and should be compensated for via a robust design of the observer feedback.

\section{IMPLEMENTATION}

The field test of the model-based molten metal analysis estimation system (SafeCon) are performed at the plant in Luleå . In order to reduce implementation time for the test series, SafeCon is installed on the same process computer as MEFCON. Thereby, SafeCon can exploit MEFCON's data acquisition routines.

Since SafeCon is not integrated with the converters control system, the correct handling of events and initialisation of the algorithm is very important for the implementation.

\section{A. Process computer}

MEFCON is installed on a Compaq Alphastation running OpenVMS as operating system. Fig. 1 shows the structure of the implementation, signal flow and how SafeCon accesses the acquired process data.

In Fig. 1 it can be seen that MEFCON and SafeCon have access to the same common area, which is updated with process data by data acquisition processes and by both MEFCON and SafeCon. The common area not only contains raw data, it can also store preprocessed data and act as a transfer buffer between processes.

Here, SafeCon uses the common area to acquire raw data from the process and preprocessed data from MEFCON, which are mainly flags that indicate events in the LD converter. Furthermore, the generated estimates are written back to the common area and are presented to the operators via the human machine interface.

SafeCon itself is running as an asynchronous system process with a fixed cycle time (one second). In order to give the user a minimum degree of interaction with SafeCon a configuration file is used, which is read by SafeCon before a new heat is started. Consequently, changes in the configuration take effect without restarting SafeCon.

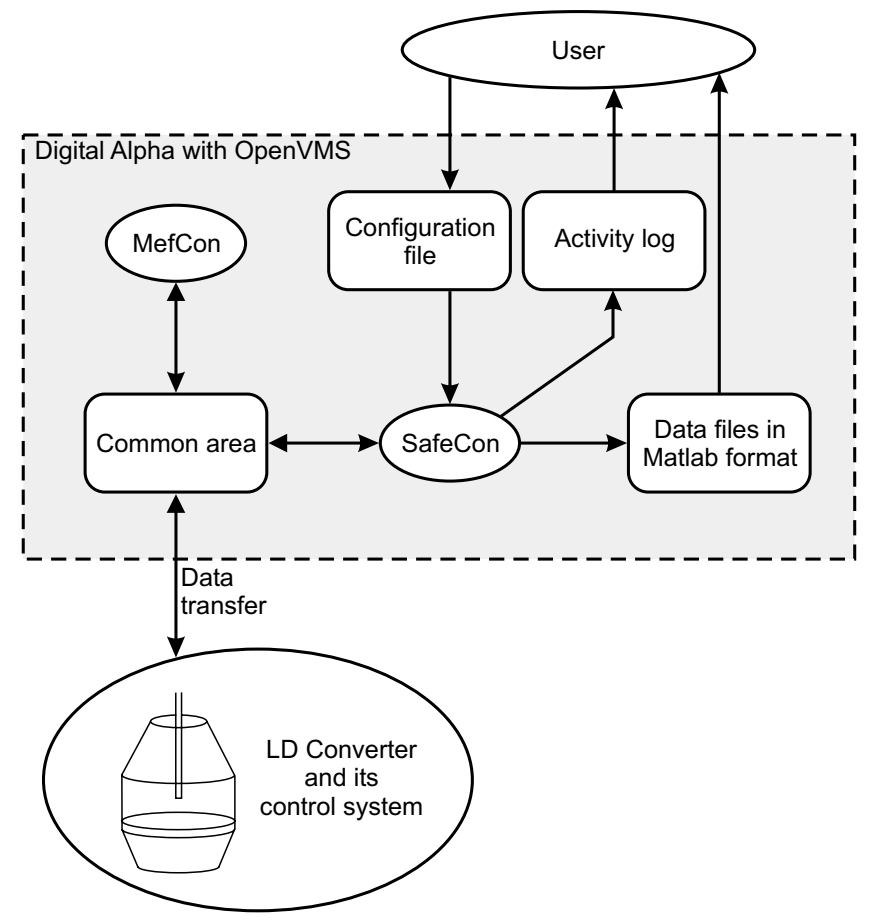

Fig. 1. Implementation of Safecon.

During a heat, SafeCon acquires external data from the LD Converter process, generates internal data, i.e. process state information, and generates output data, i.e. molten metal analysis in real time. These data and additional information on the heat are written to files in Matlab format. Each heat is assigned to a separate file. The data files can then be accessed and analyzed by the user.

Moreover, SafeCon generates an activity log, which contains time stamped information on all activities.

The data files are formatted so that a simulation environment on a PC computer can directly access the data of a heat and simulate it. Hence, debugging, re-design and refinement of SafeCon are facilitated.

\section{B. Events}

Since SafeCon is implemented as a continuously running process an event-driven state machine has to be designed that detects key events and changes the state of the estimator.

Key events are:

- Start of a heat

- End of a heat

- Interruption of a heat

- Restart of a heat

- Introduction of additive

By combining data from the common area and detecting flanks in the data, all key events can be detected. Still, some events do not occur instantaneously, whereby a definition of the time of occurrence becomes uncertain, e.g. Start of a heat.

When a heat is started, the oxygen is turned on when the lance is not yet in place. Consequently, decarburisation starts before 
the final lance position is reached. Assuming that decarburisation during the early beginning of a heat is negligible, the time of occurrence of this event is given as both final lance position is reached and oxygen flow is switched on.

In case of the End of a heat the situation is not that crucial, as the estimator freezes in its current states, when the oxygen flow is switched off. Thus, a prolonged attendance of the estimator is not of any harm and the event occurs when the converter is tilted for tapping.

Moreover, this definition of End of a heat facilitates the detection of Interruption of a heat or Restart of a heat. An interruption often occurs in the early stages of a heat and can be caused by slopping, malfunction or a congestion in the steel casting plant. Whereas, a restart is triggered when the operators have not reached the desired final carbon content.

The event Introduction of additive is well defined in time and does occur abruptly. Thence, a detection from data is an elementary task.

\section{Initialisation}

When Start of a heat is detected, SafeCon should be initialised with the initial conditions of the newly charged converter. Important contiguities are hot metal weight and content of silicon and carbon. Due to varying time delays in the metal analysis of the hot metal, these contiguities may not be available at the start, which prevents the correct initialisation of SafeCon. But, because of the feedback in the estimation algorithm, deviations in the initial values do not necessarily lead to errors in the final estimate.

Thus, if the hot metal analysis is delayed a prediction of the analysis, which is rather coarse, is used. An immediate consequence is a loss of estimation accuracy and converter operators have to be informed. In a later version, SafeCon should enter a data buffering mode, where the data is stored in a FIFO buffer in real time. As soon as the analysis arrives SafeCon continues to buffer but also spawns a non-synchronised sub-process that simulates the heat. When finally all buffered data is processed, SafeCon returns from the buffering mode to the standard mode, where the estimator runs online in real time again.

Moreover, initialisation must not occur after Interruption of a heat or before Restart of a heat, as it would devastate the estimation result.

\section{Restart}

When Restart of a heat takes place, the final carbon content in the hot metal is not close enough to the desired one. Usually, the operators initiate a new shorter heat with the same load in the converter.

SafeCon has to disregard the restart and has to freeze its estimate during the renewed heat.

\section{E. Additives}

An often used practice of the LD converter operators is to add additives to the metal bath in the final stage of the heat.

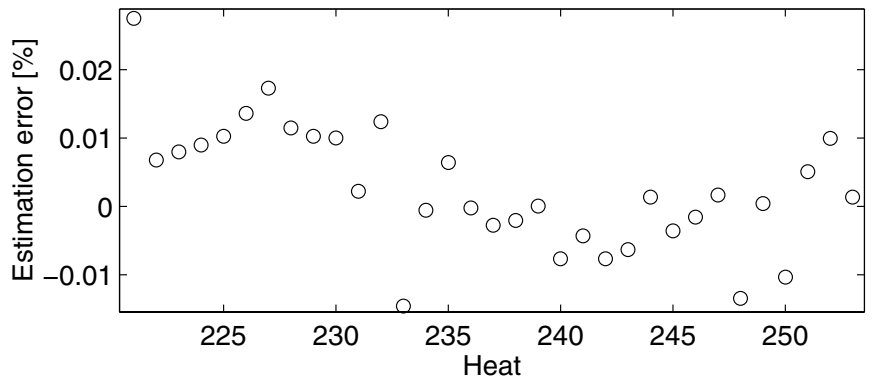

Fig. 3. Estimation error over time

Sometimes additives are supplied to the process even after the end of the heat.

Then, deviations between estimate and molten metal analysis come about. The effect of additives on the behavior of the estimate has yet to be studied.

Currently, no bounds for estimation accuracy are given. This should be provided for the operators in a later version to improve reliability.

\section{PRELIMINARY RESUltS}

Fig. 2 shows the results from all heats without late additives between January and April 2001 at the plant in Luleå. Included in the figure is also a normal distribution with the same standard deviation for reference. Clearly, the distribution of the estimation error is not completely random, which indicates that improvements are possible.

Note for example the high representation of positive errors between $0.005 \%$ and $0.015 \%$. One possible explanation for this is due to the observer structure where the feedback is weighted by the sensitivity of the output with respect to the carbon and silicon contents. If the estimated carbon content is too high during the blow, then the feedback will be too weak which leads to slower convergence compared to the case when the estimated carbon content is too low.

Fig. 3 shows the errors for a number of heats. There is obviously some correlation from one heat to the next. This can be utilized by predicting the error and thereby reducing it.

\section{A. Heat operators}

From Fig. 4 (lower part) it is evident that there are differences in the quality of the estimate, depending on which operator team that runs the converter process.

There appears to be a negative correlation between how well the final carbon content is achieved by the operators (upper plot) and how well the algorithm performs. This is however dependent upon the values of the observer parameters. The main conclusion is that there seems to be some difference in procedure between the teams, which is reflected in different behaviour of the process.

The number of blows for each team is between 30 and 100 in the data for Fig. 4 and thus the difference between the teams 


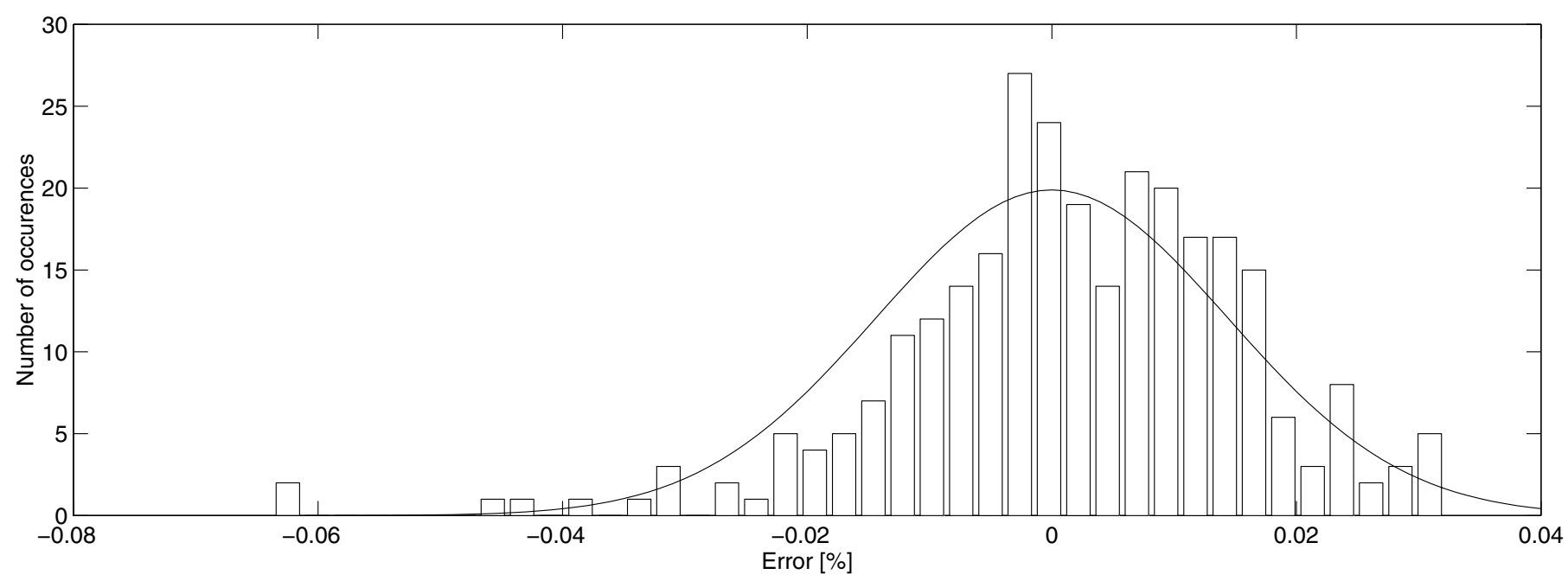

Fig. 2. Results from the heats between January and April 2001
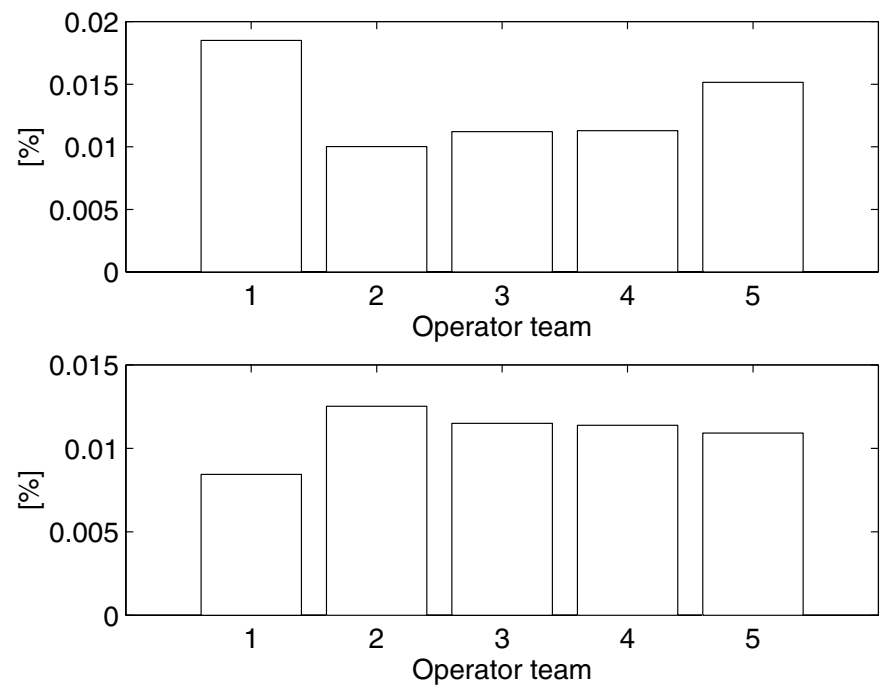

Fig. 4. Difference between operator teams. Upper plot shows mean error in achieved carbon content, while lower plot shows mean estimation error of the observer

are assumed to be statistically guaranteed. Differences between individual operators cannot be determined due to insufficient data.

\section{B. Bath level}

One possible cause of the estimation error are variations in the distance between the lance and the metal surface, caused by variations in the bath level, i.e. the level of the liquid metal surface. This distance affects the impact area of the oxygen jet from the lance, and thus has a direct influence on the decarburization rate. Since the growth of foaming slag is also dependent on this distance, and the foam volume is important for the decarburization process, there is also an indirect influence from

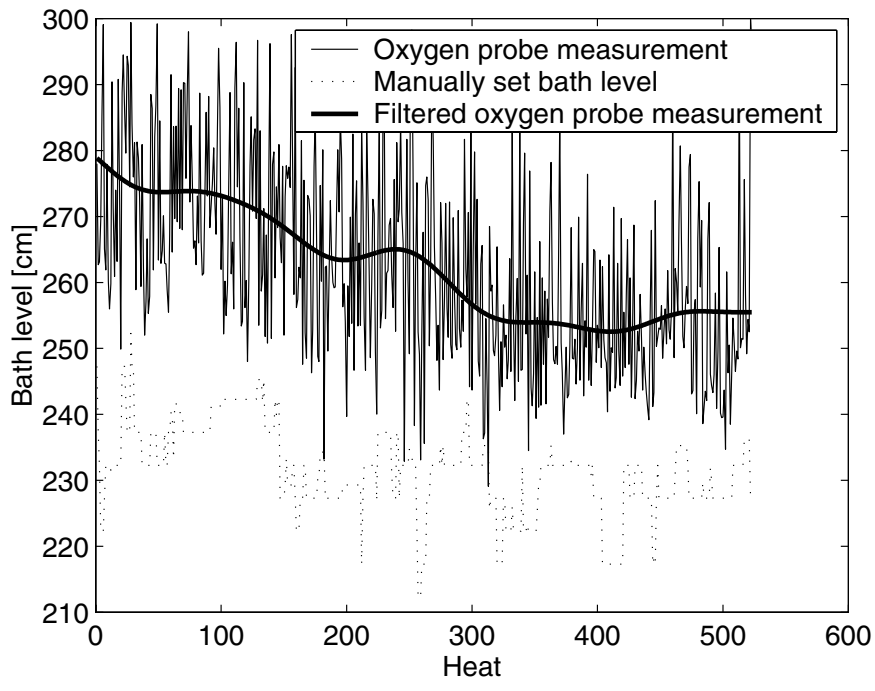

Fig. 5. Variations in bath level

the bath level on the decarburization rate.

Fig. 5 shows measurements of the bath level using an oxygen probe. This measurement is regarded as unreliable and noisy, but its filtered counterpart shows that the signal indeed carries information about the bath level. This can be seen by noting that the bath level seems to have fallen $20 \mathrm{~cm}$ during 500 heats, which is roughly the expected value due to wear of the converter lining.

The operator personnel makes an estimate of the bath level, based upon a number of factors, including measurements of the composition of the slag. This estimate, shown in Fig. 5 as 'manually set bath level', is used as the true bath level, when calculating the absolute lance height, to obtain a certain distance between lance and metal surface. It is not clear how reliable this 


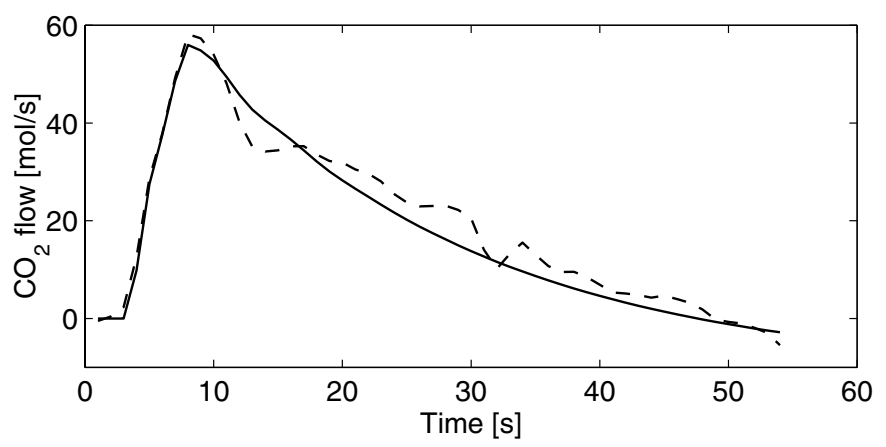

Fig. 6. Response in off-gas flow of $\mathrm{CO}_{2}$ when adding $600 \mathrm{~kg}$ of dolomitic lime. Dashed line shows the measured response while the solid line is the simulated response of a 4:th order identified linear model

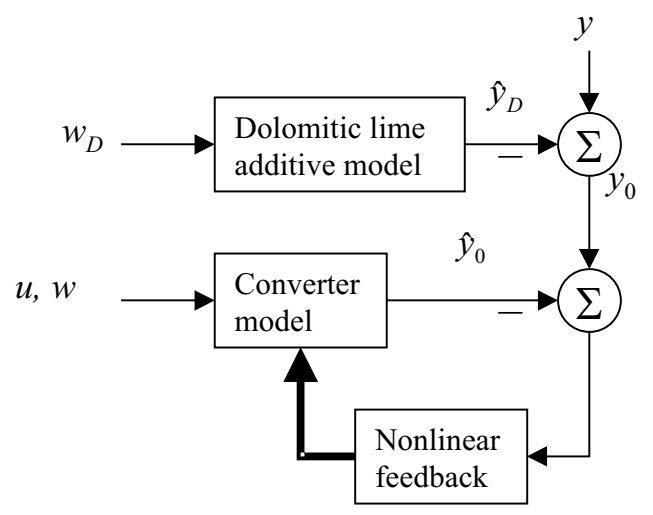

Fig. 7. Static compensation for the effects of dolomitic lime additives.

estimate is and from the figure it is evident that it does not show much similarity to the bath level measurement, apart from the overall trend.

\section{FUTURE WORK}

Further research is required on how to deal with the effects of additives, in particular dolomitic lime but also ferrosilicon.

When adding dolomitic lime, it will melt and release $\mathrm{CO}_{2}$ to the liquid metal. A model for this process can be obtained by measuring the excess $\mathrm{CO}_{2}$ in the off-gases after the additive is released. Fig. 6 shows measured and simulated response in $\mathrm{CO}_{2}$ flow from a $600 \mathrm{~kg}$ addition of dolomitic lime.

The existing strategy for dealing with additions of dolomitic lime is to subtract the simulated flow of carbon $y_{D}$ due to the additive from the measured carbon flow from the converter $y$ and obtaining the decarburization rate $y_{0}$ from this difference [4]. Fig. 7 shows the principal structure of this approach, applied to the method used in this paper. Adding of dolomitic lime is represented by the input $w_{D}$ and the estimated carbon flow in the off-gases resulting from this additive is denoted $\hat{y}_{D}$. Estimated carbon flow in the off-gases due to decarburization is denoted $\hat{y}_{0}$ while $u$ and $w$ are input signals to the converter process.

There are, however, large uncertainties in the model of the

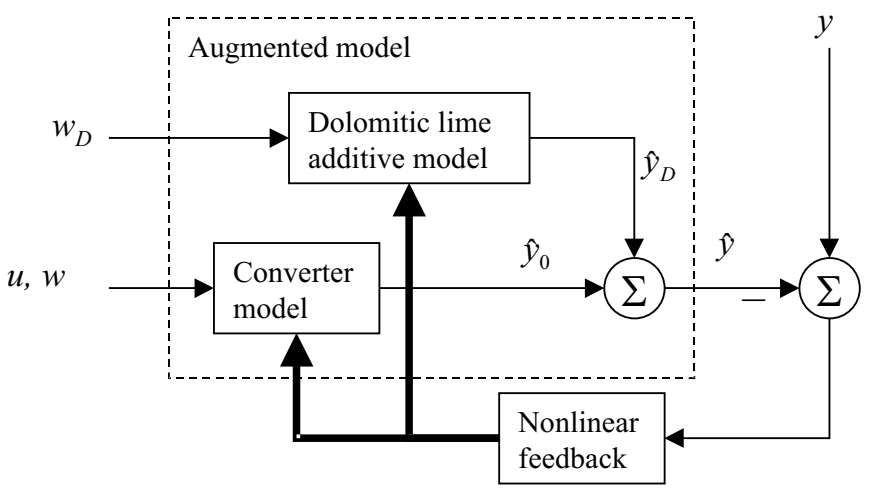

Fig. 8. Dynamic compensation for the effects of dolomitic lime additives with feedback of the output error into the dolomitic lime model

addition of dolomitic lime and therefore this static method will create disturbances in the decarburization rate fed back to the converter model.

One way of dealing with this problem is to include the model for the dolomitic lime reaction with the converter model and apply feedback to this augmented model (Fig. 8). In this context, it could also be advantageous to utilize the redundant information in the off-gas analysis to be able to separate the contribution from decarburization from that of dolomitic lime addition into the carbon flow in the off-gas.

\section{REFERENCES}

[1] A. Johansson, A. Medvedev, and D. Widlund, "Modelbased estimation of decarburization rate and carbon content in the basic oxygen steelmaking process," in Conference Record of the 2000 IEEE Industry Applications Conference, Oct. 2000. Available on CD-ROM.

[2] D. Widlund, A. Medvedev, and R. Gyllenram, "Towards model-based closed-loop control of the basic oxygen steelmaking process," in Preprints of the 9th IFAC Symposium Automation in Mining, Mineral and Metal Processing, 1998.

[3] http://www.mefos.se/mefcon.htm.

[4] D. Bergman and P. Hahlin, "Experience of waste gas analysis based control system for the LD-LBE-process at SSAB Tunnplåt AB, Luleå, Sweden," in Second European Oxygen Steelmaking Congress (EOSC '97), Taranto, Italy, Associazione Italiana di Metallurgia, Oct 1997.

[5] A. Johansson, A. Medvedev, and D. Widlund, "Modelbased estimation of metal analysis in steel converters," in 39th IEEE Conference on Decision and Control, pp. 20172022, December 2000. 